Ubiquitin in Regulation of Spindle Apparatus and its Positioning: Implications in Development and Disease

\begin{tabular}{|r|l|}
\hline Journal: & Biochemistry and Cell Biology \\
\hline Manuscript ID: & bcb-2015-0011.R1 \\
\hline Manuscript Type: & Mini Review \\
\hline Date Submitted by the Author: & 01-May-2015 \\
\hline Complete List of Authors: & $\begin{array}{l}\text { Srivastava, Devika; Saha Institute of Nuclear Physics, Biophysics \& } \\
\text { Structural Genomics } \\
\text { Chakrabarti, Oishee; Saha Institute of Nuclear Physics, Biophysics and } \\
\text { Structural Genomics }\end{array}$ \\
\hline Keyword: & $\begin{array}{l}\text { mitosis, ubiquitination, spindle positioning, cortical regulation, spindle } \\
\text { integrity }\end{array}$ \\
\hline & \multicolumn{2}{|l}{} \\
\hline
\end{tabular}

SCHOLARONE ${ }^{m}$

Manuscripts 


\title{
Ubiquitin in Regulation of Spindle Apparatus and its Positioning: Implications in Development and Disease
}

\author{
Devika Srivastava, Oishee Chakrabarti \\ Biophysics and Structural Genomics Division, Saha Institute of Nuclear Physics, Kolkata, \\ India.
}

Correspondence: O Chakrabarti, Biophysics and Structural Genomics Division, Saha Institute of Nuclear Physics, Sector-1, Block-AF, Bidhannagar, Kolkata, West Bengal 700064, India. Tel: +91 332337 5345; Fax: +913323374637

E-mail: oishee.chakrabarti@saha.ac.in

Key words: Mitosis, ubiquitination, spindle positioning, cortical regulation, spindle integrity. 


\begin{abstract}
Emerging data implicates ubiquitination, a post-translational modification, in regulating essential cellular events -- one of them being mitosis. In this review we discuss how various E3 ligases modulate the cortical proteins, like, dynein, LGN, NuMa, G $\alpha$ along with polymerization, stability, and integrity of spindles. These are responsible for regulating symmetric cell division. Some of the ubiquitin ligases regulating these proteins include PARK2, BRCA1/BARD1, MGRN1, SMURF2 and SIAH1; these play a pivotal role in the correct positioning of the spindle apparatus. A direct connection between developmental or various pathological disorders and the ubiquitination mediated cortical regulation is rather speculative, though deletions or mutations in them lead to developmental disorders and disease conditions.
\end{abstract}




\section{Introduction}

One of the earliest events that occur in a cell, post fertilization is rapid cell division. It serves two purposes - (i) proliferative function to increase in cell number and (ii) gives rise to cellular diversity in the growing organism. While the former is taken care of by symmetric cell division, asymmetric cell division serves to generate cells of different lineages. Errors that are incorporated during this stage get permanently incorporated in the cell. Aberrations during cell division can lead to multiple problems which range from developmental anomalies, defective tissue organization and even uncontrolled cell proliferation leading to cancer. Cell division is under complex regulation from multiple players such as DNA compaction, regulation of cyclin/CDKs and check points. Another important determinant of cell division which ensures normal growth and development of an organism, and simultaneously also determines the future of daughter cells is the axis of cell division (Gonczy 2008; Morin and Bellaïche 2011; Gillies and Cabernard 2011).

Orientation of cell division establishes the content, position, and fate of daughter cells within tissues. First, this helps generate elongated cell sheets and define tissue shapes. Secondly, it determines cellular diversity. In order to achieve these two mutually nonexclusive functions, the orientation of the mitotic spindle needs to be controlled. Oscar Hertwig first recognized that spindle orientation and cell division orientation were instrumental in determining cell shape; he further discovered that cells divide along their long cell axis, an observation known as the 'long axis rule' (Hertwig 1984). Symmetric cell division refers to the equal partitioning of cellular constituents, giving rise to two identical daughter cells. On the other hand, asymmetric cell division gives rise to two non-identical daughter cells with unequal partitioning of cellular constituents.

Depending on their spatial orientation, shape, structure and function, cells may be broadly classified into two categories - polarized and non-polarized cells. During the early stages, post fertilization in mammals, rapid cell division is observed. These cells are pleuripotent stem cells which undergo symmetric cell division to give rise to clonal population of cells. At some point during this clonal expansion, polarity is established using external and internal cues. Post this stage, the stem cells undergo both, symmetric division (for self-renewal) and asymmetric cell division (to give rise to differentiated cells of various lineages) (Fig 1a). The differentiated cells may be of polarized or non-polarized in nature (Gönczy 2008; Knoblich 2008; Morin and Bellaïche 2011; Gillies and Cabernard 2011; 
Williams and Fuchs 2013; Lu and Johnston 2013). Non-polarized cells typically undergo symmetric cell division which serves to maintain cell number and homeostasis in an organism (Siller and Doe 2009). However, under the influence of external cues or improper spindle positioning, these might be able to divide with unequal partitioning of cellular constituents (i.e. asymmetric cell division) (Lancaster and Baum 2011) (Fig 1b). In case of polarized cells, the axis of division becomes a defining feature for the shape and characteristics of the daughter cells generated. Cells divide along the apico-basal axis to give rise to cells which belong to different niches. At an organismal level, this acts as one of the driving forces that guide the formation of the neural tube or alimentary canal from the polarized neuronal or epithelial cells. Polarized cells can also undergo division along the anterior-posterior axis and this division is governed by planar cell polarity (PCP pathway) (Simons and Mlodzik 2008; Devenport 2014; Morin and Bellaïche 2011) (Fig 1c). A battery of proteins and their gradients define the plane of the axis of division. These proteins determine the position and plane of divisional axis, which in turn gives rise to symmetric or asymmetric cell division. Both these divisions occur in case of polarized cells, based on external and internal cues. While the discussion on the effect of external cues is beyond the scope of the present discussion, the internal cues regulating the same are discussed briefly in this section.

Central to the machinery that regulates cell division axis and spindle positioning are partitioning family cell polarity regulator proteins. These determine the axis of division and gradient of proteins governing the same. Besides these, there are a host of cortically enriched proteins that assist in the correct division by translating the external and internal cues into cortical forces. These molecular interactions at the cell cortex in turn translate into the force necessary to hold the spindle apparatus in place and along the thus determined axis of division. The force required to stabilize the spindle apparatus in correct position and orientation, is generated by the interaction of astral microtubules with the cortical proteins and minus end directed motor protein, dynein. Dynein is recruited to the membrane by the help of an evolutionarily conserved ternary complex of proteins consisting of NuMa, LGN and $\mathrm{G} \alpha$-subunit of the trimeric G-protein complex (Lin-5/GPR-1/2/G $\alpha$ respectively in worm and Mud/Pins/Go in flies) (Du and Macara 2004; Siller and Doe 2009; Stevermann and Liakopoulos 2012; McNally 2013). Dynein serves as an anchor to maintain a connection with the depolymerizing microtubule, which then exerts the force necessary to position the spindle apparatus (Kotak et al. 2013) (Fig 1d). The details and intricacies of this process are 
discussed in the course of this review in the context of different mitotic defects leading to disease conditions. Recent reports suggest that proteins participating in this regulation are mitotic motor protein KIF15 (Kinesin superfamily protein), Eg5 (also known as KIF11) (van Heesbeen et al. 2014), certain RanGTP and CLASP (Cytoplasmic linker associated protein 1) proteins (Bird et al. 2013). While CLASP1 is required for correct capture of astral microtubules, RanGTP and PLK1 ensure that the ternary complex is restricted to only the cell cortex and not the membrane area in vicinity of the metaphase plate. RanGTP has also been shown to promote astral microtubule elongation along the long axis of cells in Xenopus egg extracts (Zhang et al. 1999). Another aspect that is essential for the proper functioning of these cortical regulators is the integrity of the spindles formed during mitosis. If these fail to form stable structures during mitosis, no amount of cortical regulation can ensure accurate cell division.

Our understanding of the importance of post-translational modifications that regulate cortical proteins, spindle and astral microtubule polymerization and positioning is still premature. In this review, we discuss the emerging importance of ubiquitination in modulation of the molecular players affecting the orientation of cell division, leading to pathological abnormalities and disease conditions during their functional inactivation. 


\section{Microtubule assembly regulators}

\subsection{Ubiquitin E3 ligases}

The covalent attachment of ubiquitin to specific protein substrates is achieved by the activity of E3 ubiquitin ligases. These ligases are divided into multiple classes defined by the structural element of their E3 functional activity. They are broadly classified into HECT (Homologous to E6-AP C-terminus)-family E3 ligase, and RING (Really Interesting New Gene). An extension of the RING family includes homeodomain (PHD) and leukemia associated protein (LAP) finger proteins, and members of the U-box family (a modified RING motif without the full complement of $\mathrm{Zn} 2+$-binding ligands) domain. While HECT E3s directly catalyse ubiquitination, RING and U-box E3s have been shown to facilitate this post-translational modification by primarily acting as scaffold proteins (Metzger et al. 2012). Both HECT and RING ligases transfer activated ubiquitin from a thioester to the $\varepsilon$-amino acid group of a lysine residue on the substrate. HECT ligases have an active site cysteine that forms an intermediate thioester bond with ubiquitin, on the other hand RING ligases interact with the ubiquitin conjugating enzymes to allow direct ubiquitin transfer from the E2 to substrate. Another family of ligases is the RBR family (Ring-Between-Ring) of proteins that function with a canonical RING domain and a catalytic cysteine residue usually restricted to HECT E3 ligases. Hence these ligases are also known as 'RING/HECT hybrid' enzymes (Wenzel et al. 2011; Stieglitz et al. 2012).

\subsubsection{PARK2}

Parkin (PARK2), an RBR ligase is widely implicated in the onset and progression of Parkinson's disease. Numerous reports suggest the dysregulation of the ubiquitin proteasome system (UPS) in the pathogenesis of Parkinson's disease (Hristova et al. 2009).

Recent studies show a more detailed and direct influence of PARK2 on cell division (Sun et al. 2013). Studies with pancreatic tumor samples from patients exhibit reduced expression levels of PARK2. Various mitotic defects were detected in these cases, vis-à-vis multiple spindle formation, chromosomal instability, spindle tilt along the axis of division and increase in the vertical distance between the spindle poles. These observations could be replicated in pancreatic cancer cell line, EPP85 with the depletion of PARK2. These 
observations indicate an obvious involvement of PARK2, direct or indirect, in cell division (Sun et al. 2013).

It has been hypothesized that PARK2 modulates proteins critical for mitotic spindles via its regulation of the microtubule motor protein, Eg5 expression (Sun et al. 2013). Eg5 is a bipolar, plus-end-directed microtubule motor protein (Tanenbaum and Medema 2010). Depletion or inhibition of Eg5 activity prevents bipolar spindle formation and arrests cells in mitosis with unseparated centrosomes. Eg5 can crosslink microtubules into an antiparallel configuration and slide them apart, thus generating an outward-directed pushing force on centrosomes (Kapitein et al. 2005; Tanenbaum et al. 2009). Under normal cellular context, it has been shown that PARK2 mediates multi-monoubiquitination of Hsp70 which inactivates c-Jun NH2-terminal kinase, resulting in decreased phosphorylation of c-Jun. This leads to reduced c-Jun binding to the activator protein 1 site present in the Eg5 promoter and downregulation of Eg5 gene transcription (Liu et al. 2008) (Fig 2). Hence, under conditions when PARK2 ligase activity is repressed, Eg5 activity is indirectly elevated resulting in aberrant mitotic apparatus.

However, the role of $\mathrm{Eg} 5$ in cell division is contentious. Eg5 has been demonstrated to participate in prophase centrosome separation in mammalian cell systems (Blangy et al. 1995; Sawin and Mitchison, 1995; Whitehead et al. 1996), while in Drosophila embryos, its homolog does not have a similar role in prophase (Sharp et al. 1999). Moreover, even in mammalian systems, prophase centrosomes have been suggested to move independently of each other without the involvement of Eg5 (Waters et al. 1993). Hence, the importance/function of $\mathrm{Eg} 5$ in bipolar spindle assembly is far from clear.

Thus, though there are evidences for the regulation of spindle tilt by PARK2, the exact mechanism still needs to be clearly understood. It is plausible that PARK2 regulates spindle tilt and pole formation in an Eg5 independent manner.

\subsubsection{BRCA1/BARD1}

Germline mutations in Breast cancer-associated gene 1 (BRCA1) have been associated with familial breast and ovarian cancers. Further, downregulation of BRCA1 has been reported in sporadic breast and ovarian cancers. BRCA1 undergoes heterodimerisation with BRCA1-associated RING domain protein, BARD1 (Wu et al. 2006). The RING 
domains of BRCA1 and BARD1 form a dimerization interface and the BRCA1/BARD1 heterodimer functions as an ubiquitin ligase (Parvin 2009). This has been shown to participate in cell proliferation and chromosome stability.

Homozygous Brcal knockout mice die in utero between 10 and 13 days of gestation (E10-E13). These mice have abnormalities in the neural tube, with $40 \%$ of the embryos showing varying degrees of spina bifida and anencephaly. Further, the neuroepithelium in Brcal-deficient embryos also seem to be disorganized (Parvin 2009). At the cellular level, BRCA1 and BARD1 localize to the centrosome during the cell cycle (Sankaran et al. 2005). Embryonic fibroblasts from Brcal knockout mice show centrosome amplification and genome instability (Kais et al. 2012). BRCA1 is known to bind to $\gamma$-tubulin, a major component of centrosomes. Studies in Xenopus egg extracts and HeLa cells show that this BRCA1/BARD1 complex then mono-ubiquitinates $\gamma$-tubulin on Lysine 48 (K48) and K344 (Joukov et al. 2006). The BRCA1/BARD1 ubiquitin ligase activity regulates centrosome number and function. Further, BRCA1/ BARD1-dependent ubiquitination activity modulates the microtubule nucleation and centrosomal hypertrophy (Sankaran et al. 2005). Cells with depleted BRCA1/BARD1 show suppressed aster formation in interphase (Tarapore et al. 2012). BRCA1/BARD1 is also required for mitotic spindle pole assembly and for the accumulation of TPX2 (targeting protein for Xklp2), a major spindle organizer and a downstream target of Ran-GTP, on spindle poles. This function is centrosome independent, operates downstream of Ran GTPase, and depends upon BRCA1/BARD1 E3 ubiquitin ligase activity (Daniels et al. 2004) (Fig 3).

As indicated before, the microtubule aster polymerization and spindle pole assembly is an important player that regulates the positioning of the spindle apparatus in the center of the cell, along the planar axis of division during in case of polarized cells. These observations about BRCA1/BARD1 can thus be extrapolated to explain the increased propensity for breast cancer in individuals who harbor mutations in these genes.

BRCA1/BARD1 is known to localize to centrosomes during metaphase where this ubiquitinates and degrades Aurora B (Ryser et al. 2009). The same study has elucidated that various cancer cells express RING-deficient isoforms of BARD1, called BARD1 $\beta$. This isoform can have BRCA1-independent functions (Ryser et al. 2009). It has been shown that BARD1 $\beta$ localizes to the midbody at telophase and cytokinesis, where it associates with and stabilizes Aurora B, a protein crucial for midbody formation and abscission (Delaval et al. 
2004; Ryser et al. 2009). Thus, the elevated levels of BARD1 $\beta$ and the imbalance between the BARD1/BARD1 $\beta$ levels in cancer cells could partially contribute to their increased rate of cell division.

\subsubsection{VHL}

von Hippel-Lindau disease, an inherited cancer syndrome is associated with an E3ligase, VHL (von Hippel-Lindau tumor suppressor) in which patients are predisposed to develop various vascular tumors and clear cell renal cell carcinomas (ccRCC) highlighting distinct roles for VHL in tissue morphogenesis and tumorigenesis (Kaelin 2007). It is the substrate recognition component of the cullin-RING ubiquitin ligase complex (CRL) that includes cullin-2, Rbx 1, and elongins B and C (Deshaies and Joazeiro 2009; Kaelin 2008). VHL is best characterized for its role as a key component of an E3 ubiquitin ligase that targets hypoxia-inducible factor (HIF) for degradation (Kaelin 2008; Jaakkola et al. 2001). The homozygous knockout of $V h l$ leads to embryonic lethality at E14.5 to E18.5. Heterozygous knockout mice however, appear normal in their development, growth and reproductivity (Zhang et al. 2012).

VHL is known to play a role in spindle orientation as its knockdown in MEFs and HeLa causes spindle misorientation by eliminating astral microtubules. The defects observed by VHL downregulation bear striking resemblance to that of APC (Adenomatous polyposis coli) inactivation which includes compromised spindles and misaligned chromosomes (Thomma et al. 2009). One of the possible reasons might be that VHL positively regulates MAD2 (mitotic arrest deficient 2) levels in cells (Thoma et al. 2009; Liu et al. 2011). MAD2 is a member of mitosis checkpoint complex at the kinetochore, where it interacts with other proteins like Bub1 (Budding uninhibited by benzimidazoles 1 homolog). MAD2 reduction is seen to cause aneuploidy in cells, an observation widely prevalent in VHL-defective cells as well. This is consistent with the known contribution of VHL to microtubule stability and cell cycle regulation. Further, VHL may be required for the degradation of incorrectly folded tubulin, suggesting that VHL could also contribute to MT dynamics (Delgehyr et al. 2009). 


\subsubsection{MGRN1}

Mahogunin ring finger-1 (MGRN1) was identified by positional cloning of mahoganoid (md) (Miller et al, 1997), a mutation characterized by darkening of coat color in mice. Mutant mice show changes in the levels of expression and activity of mitochondrial proteins in the brain which precedes the development of spongiform neurodegeneration with many features of prion diseases (Cota et al. 2006; Jiao et al. 2009). Although the mechanisms leading to spongiform neurodegeneration are not fully understood, data shows that some of the disease causing abnormal isoforms of $\operatorname{PrP}$ (transmembrane isoform, ${ }^{\mathrm{Ctm}} \operatorname{PrP}$ and cytoplasmic isoform, cyPrP), can engage in atypical interactions with MGRN1 (He et al. 2003; Kim et al. 2007; Chakrabarti and Hegde. 2009). Independent studies indicate that while nearly 50\% of Mgrn1-null mutants in mice develop late-onset spongiform neurodegeneration, rest exhibit severe developmental defects (like congenital heart defects, abnormal craniofacial patterning and mispatterning of the left-right (LR) body axis) and increased mortality by weaning age, thus suggesting multiple roles for MGRN1. Additionally, about 1\% of animals also suffer from situs inversus (complete reversal of the left and right body axes) (Cota et al. 2006; Jiao et al. 2009).

Recent study from our laboratory shows that $\alpha$-tubulin is a substrate for MGRN1 (Srivastava and Chakrabarti 2014). It is shown to polyubiquitinate $\alpha$-tubulin and promotes its polymerization. This report further shows that in cell culture systems (HeLa and MEFs), mitotic cells which over-express functionally inactive forms of MGRN1 exhibit multiple spindle defects ranging from spindle tilt and shortening of aster microtubules. It is possible that the poorly formed spindles cause an inherent instability in the spindles. These malformed spindles may not engage in robust interactions with cortical proteins, leading to the observed defects. It would be worth exploring if MGRN1 regulates other players to affect the spindle positioning. Unpublished data from our laboratory shows that MGRN1 modulates the cortical enrichment of the proteins involved in spindle positioning (Fig 4). These studies give us an attractive handle to study and dissect the activity of MGRN1 in cell culture systems and explore the cause of the pleiotropic effects observed in its absence during embryonic development. The modulatory effect of MGRN1 on the microtubules may not, however, directly affect spongiform neurodegeneration. 


\subsubsection{SIAH1}

Besides the above discussed E3-ligases there are several other ubiquitinating enzymes that regulate spindle positioning and thus might be potential players in cancer. The human homologue of the Drosophila seven in absentia (Sina) (SIAH1 and SIAH 2), have been implicated in ubiquitin-mediated proteolysis of different target proteins and are known to regulate HIF $\alpha$ protein stability. SIAH1 and SIAH2 bind Propyl Hydroxylases (PHD1 and PHD3) and target them for proteasomal degradation (Nakayama et al. 2004). Double knockout mice for Siah1 and Siah2 are not viable, but single knockouts are able to live. Growth in Siahl knock-out mice is severely retarded and along with poor bone formation (House et al. 2009). Also, Siahl null mice have poor viability and display a meiotic block at metaphase I that impairs spermatogenesis and causes sterility in male mice (Dickens et al. 2002).

Murine Siah-1 is a p53-induced gene; its human homolog, SIAH-1 is activated during tumour suppression and p53 induced apoptosis. When overexpressed, it has been shown to inhibit cell growth by causing defects in mitosis and increasing apoptosis in breast cancer cell line, MCF-7. Mitotic defects include anomalies of the mitotic spindle and abnormal chromosomal segregation, multinucleate giant cells along with failure of cytokinesis. Established targets of SIAH1 include $\alpha$-tubulin and Kid (kinesin like DNA binding protein, also known as KIF22), identified by yeast two hybrid assays (Germani et al. 2000). While the significance of interaction with $\alpha$-tubulin is mostly speculative, it has been shown to ubiquitinate and degrade Kid. Kid has been proposed as a linkage regulator of chromosome movement along microtubules during mitosis. Reports showed that the Xenopus homologue of Kid (Xkid) is the motor protein that provides the necessary force to push chromosome arms toward the equator of spindle. Its degradation is necessary for anaphase chromosome movement. These observations provide a plausible explanation for the mitotic anomalies observed in the absence of SIAH1 (Antonio et al. 2000; Funabiki and Murray, 2000; Germani et al. 2000).

Interestingly, SIAH1 is also shown to polyubiquitinate microtubule plus-end tracking proteins (+TIP) binding protein EB3 (End Binding protein 3), and mediate its degradation through the ubiquitin-proteasome system. EB3 has been shown to facilitate cell cycle progression at prometaphase, and is downregulated during the transition to G1 phase in cell 
culture based studies (Ban et al. 2009). Thus, the interaction between SIAH1 and EB3 could be used as an alternative explanation for the observed mitotic defects and spindle anomalies.

\subsubsection{SMURF2}

Another interesting ligase is SMURF2 (Smad specific ubiquitin regulatory factor 2) which is a HECT E3 ligase, originally characterized as a negative regulator of the TGF- $\beta$ signaling pathway by targeting receptors, signaling intermediates, and other pathway-specific transcription factors for degradation ( Tang et al. 2011). Smurf2-/- mice (>99\%) do not exhibit any pronounced developmental defects during embryogenesis and they are phenotypically indistinguishable from their wildtype or heterozygous littermates at weaning (Lin et al. 2000). Surprisingly mice mutant for both Smurf1 and Smurf2 display planar cell polarity (PCP) defects in the cochlea and abnormalities in convergence and extension movements (CE), which include a failure to close the neural tube. Further, it has also been illustrated that SMURFs are involved in non-canonical Wnt signaling pathway via ubiquitinmediated degradation of the core PCP protein Prickle1 (Narimatsu et al. 2009).

SMURF2 exhibits a dynamic localization pattern throughout mitosis, moving from centrosomes in late prophase and metaphase, to the mitotic midzone in anaphase, and ultimately to the midbody in telophase. Study shows that SMURF2 participates in posttranslational control of MAD2 (mitotic arrest deficient 2), stabilizing the same. SMURF2 depletion results in enhanced polyubiquitination and degradation of MAD2. MAD2 mislocalizes in SMURF2-depleted cells, suggesting that SMURF2 regulates the localization and stability of MAD2, thus regulating spindle check point. In addition to its role as a regulator of the spindle checkpoint, the localization of SMURF2 to other critical mitotic structures implies its participation in other aspects of mitotic control. Further studies would be required to establish this hypothesis (Osmundson, 2004).

\subsubsection{TRIM E3 ligases}

A family of E3 ligases worth mentioning is TRIM family (The conserved arrangement of a RING-finger domain, B-box, and coiled-coil domains). Several members of this family of ligases have been implicated in disease conditions namely, MID1 (Midline-1), TRIM5 $\alpha$ 
(Tripartite motif protein 5-alpha) and Xnf7 (Xenopus nuclear factor 7). While TRIM5 mediates resistance to the human virus in rhesus monkey cells (Stremlau et al. 2004), MID1 is implicated in developmental disorder, Opitz Syndrome, an inborn error of cholesterol synthesis (Cox et al. 2000).

Xnf7 (Xenopus nuclear factor 7), discovered from Xenopus egg extracts as an inhibitor of the anaphase promoting complex (APC), is a maternally expressed nuclear protein that is retained in the cytoplasm from oocyte maturation until the midblastula transition (MBT) and is shown to be involved in dorsal/ventral patterning (El- Hodiri, 1997b). Xnf7 has not yet been implicated in disease condition. Although both MID1 and Xnf7 have E3 ligase activities, their roles in governing spindle dynamics do not involve this enzymatic activity. These proteins bind to microtubules and drive their bundling, thus acting as key players in the regulation of spindle dynamics (Maresca et al. 2005). 


\subsection{Other UPS associated proteins regulating spindle dynamics}

\subsubsection{HUNTINGTIN (HTT)}

Huntingtin (HTT), implicated as the foremost player in the onset and progression of the severely debilitating neurodegenerative disease, Huntington's disease (HD), is also suggested to modulate spindle assembly and orientation. HD is a neurodegenerative disorder characterized by severe psychiatric, cognitive, and motor defects and selective neuronal death in the brain, caused by hungtintin (HTT) gene. Although the mechanisms leading to disease are not completely known, increasing evidences indicate that in addition to the gain of new toxic properties, loss of wild-type HTT function also adds to pathogenesis (Cattaneo et al. 2005). Various studies in cell models and with patient samples have reported that the UPS is impaired in HD, which could be the underlying cause of the neurotoxicity.

$H t t$ is widely expressed in the early embryonic stages in mice where it plays an important role in several processes including cell differentiation and neuronal survival. Inactivation of the mouse gene results in developmental retardation and embryonic lethality at E7.5 (Nasir et al. 1995; Zeitlin et al. 1995). Studies have now conclusively shown that HTT is involved in neurogenesis and brain development (Godin et al. 2010).

Reports suggest that HTT is ubiquitinated and it also interacts with a ubiquitin conjugating enzyme E2 (Kalchman et al. 1996). The ubiquitinated species detected may be parts of either processed or partially degraded forms of HTT. Studies in SHSY5Y cells show that stable expression of long polyglutamine tracts lead to cell cycle arrest and inhibition of proteasomal degradation pathway. The impaired proteasomal activity does not elevate protein aggregation under basal conditions, however it severely compromises the ability of the proteasome to respond to stress leading to increased stress-induced protein aggregation. This is further accompanied by altered expression of the 20 s proteasomal subunits (Ding et al. 2002).

Recent studies have shed a lot of light on the function of HTT in the cell. Wild type HTT is shown to interact with microtubules, the dynein/dynactin complex and kinesin to regulate the microtubule-dependent transport of organelles in neurons (Caviston et al. 2007; McGuire et al. 2006). HTT is found in high levels in dividing cells where it associates with the centrosomal region and microtubules (Hoffner et al. 2002). Interestingly, HTT has been shown to be required in murine neuronal progenitors for appropriate spindle orientation and 
for cell fate determination (Godin et al. 2010). Also, it has been demonstrated that mutant forms of HTT disturb the cortical localization of proteins like dynein, NuMa (MolinaCalavita M et al. 2014). A study in mammary cells throws light on the mechanistic cause of the mitotic abnormalities observed earlier. This study shows that HTT is not only important for bipolar spindle assembly but also for the cortical localization of the dynein/dynactin/NUMA/LGN complex. Thus HTT may be a connecting molecular link between the dynein/dynactin and NUMA/LGN pathways ensuring the transport of the dynein/dynactin/ NUMA/LGN complex from the spindle poles to the cell cortex. HTT is known to interact with kinesin 1 (KIF5) through the Huntingtin associated protein 1 (HAP1) to promote microtubule-based anterograde vesicular transport in neurons (Colin et al. 2008; McGuire et al. 2006). During mitosis, HTT is targeted to the spindle poles due to its interaction with dynein and there it promotes the accumulation of NUMA and LGN. It has been shown that KIF5 is a molecular plus-end motor, which in association with HTT, regulates the trafficking of the dynein/dynactin/ NUMA/LGN complex along the astral microtubules to the cell cortex during mitosis. Once at the cell cortex, the dynein/dynactin/NUMA/LGN complex generates pulling forces on astral microtubules for mitotic spindle positioning in the center of the cell (Elias et al. 2014).

Yet unclear, it will be interesting to closely study the role of HTT and see if there is any direct or indirect connection between its influence on the regulation of UPS cells, the subsequently reported cell cycle inhibition and the regulation of the spindle apparatus.

\subsubsection{Deubiquitinases}

Besides the ubiquitin ligases, the deubiquitinating enzyme, ubiquitin C-terminal Hydrolase L1 (UCH L1) also plays a significant role in modulating microtubule dynamics. $\mathrm{UCH}$ L1 is a cysteine hydrolase that contains a typical active-site triad of cysteine, histidine, and aspartic acid and catalyzes hydrolysis of C-terminal esters and amides of ubiquitin. This enzyme is expressed in abundance in brain and reproductive tissues (Setsuie and Wada, 2007). Reports indicate that mutations in the UCH L1 gene have been involved with Parkinson's and Alzheimer's diseases (Betarbet et al. 2005). Recent studies indicate that $\mathrm{UCH}$ L1 is multi-functional protein: besides being a deubiquitinating enzyme, UCH L1 dimer has an ubiquitin ligase activity in vitro. It has been implicated in mitosis by studies in cell 
culture systems, where it binds to tubulin and regulates microtubule dynamics by ubiquitination (Bheda et al. 2010).

The deubiquitinating enzyme cylindromatosis (CYLD), specifically removes lysine 63 (K63)-linked polyubiquitin chains and is a regulator of microtubule dynamics (Gao et al. 2008; Harhaj and Dixit 2011). It stabilizes astral microtubules and stimulates the formation of the Dvl (Disheveled)-NuMA-dynein/dynactin complex at the cell cortex in Hela cells and mice models. The deubiquitination of Dvl enhances its interaction with nuclear mitotic apparatus, stimulating its cortical localization in association with the dynein/dynactin motor complex. Thus generates pulling forces on astral microtubules (Yang et al. 2014). These studies suggest a role for CLYD in tumor progression as it is well known that the tumor suppressor protein is mutated in familial cylindromatosis and multiple familial trichoepithelioma, genetic conditions associated with the development of skin-appendage tumors (Massoumi 2011). 


\section{Conclusion}

From the few E3 ligases discussed above, it is evident that these proteins play an important role in cell division. This review only highlights the importance of the ubiquitin E3 ligases that directly interact with or alter mitotic spindle generation, positioning and orientation (Fig 5). It is essential to point out that there is an elaborate list of other E3 ligases, like APC and Skp, Cullin, F-box containing complex (or SCF complex) which also affect cell cycle progression and mitosis, either directly by modulating the cell cycle check points or indirectly, by altering the functions of other MT interacting proteins. Surprisingly, while all the ligases discussed in this review are crucial for successful mitosis, all of them except BRCA1 and VHL are non-essential - in other words their loss of function does not lead to embryonic lethality. One probable explanation could be a phenomenon similar to what is observed in Drosophila during development -- "telophase rescue". It redistributes fate determinants in accordance with spindle orientation just before cytokinesis in the majority of mutant neuroblasts, irrespective of the $\mathrm{AB}$ polarity axis. Mutations in mud (homolog of NuMa present in Drosophila) are only able to cause defects in spindle orientations but not affect the polarity axis during division. The mechanism of "telophase rescue" are not entirely clear (Schober et al. 1999; Wodarz et al. 1999; Peng et al. 2000; Neumüller and Knoblich, 2009; Morin and Bellaïche, 2011). It is plausible to hypothesize that similar salvage mechanisms exist in mammalian systems as well, thus ensuring correct division during development. Alternatively, it also raises the possibility of functional redundancy between the isoforms of the proteins and amongst different types of proteins as well. Though it may not have been studied in depth in the context of the ubiquitin E3 ligases, this concept is not new as there exists examples of functionally redundant proteins in the genome ( Kafri et al. 2006). It has been shown that responsive backup circuits may function as devices for filtering non-genetic noise from transcriptional pathways, thus rendering an added layer of protection to the organism against stress and mutations (Massoumi 2011). More extensive genome wide studies are required to prove that similar backup circuits exist with respect to E3 ligases as they may seem functionally irreplaceable at the cellular levels, yet they are non-essential at the organismal level during development. 


\section{References}

1. Antonio C, Ferby I, Wilhelm H, Jones M, Karsenti E, Nebreda AR, et al.. 2000. Xkid, a chromokinesin required for chromosome alignment on the metaphase plate. Cell 102(4): 425-35

2. Ban R, Matsuzaki H, Akashi T, Sakashita G, Taniguchi H, Park SY. 2009. Mitotic Regulation of the Stability of Microtubule Plus-end Tracking Protein EB3 by Ubiquitin Ligase SIAH-1 and Aurora Mitotic Kinases. J Biol. Chem. 284(41): 28367-81

3. Betarbet R, Sherer TB, and Greenamyre JT. 2005. Ubiquitin proteasome system and Parkinson's diseases. Exp. Neurol. 191Suppl 1: S17-27

4. Bheda A, Gullapalli A, Caplow M, Pagano JS, and Shackelford J. 2010. Ubiquitin editing enzyme UCH L1 and microtubule dynamics Implication in mitosis. Cell Cycle 9(5): $980-94$

5. Bird SL, Heald R and Weis K. 2013. RanGTP and CLASP1 cooperate to position the mitotic spindle. Mol. Biol. of Cell 24(16): 2506-14

6. Blangy A, Lane HA, d'Hérin P, Harper M, Kress M and Nigg EA. 1995. Phosphorylation by $\mathrm{p} 34 \mathrm{cdc} 2$ regulates spindle association of human Eg5, a kinesin-related motor essential for bipolar spindle formation in vivo. Cell 83(7): 1159-69

7. Cattaneo E, Zuccato C, and Tartari M. 2005. Normal huntingtin function: an alternative approach to Huntington's disease. Nat. Rev. Neurosci. 6(12): 919-30

8. Caviston JP, Ross JL, Antony SM, Tokito M, and Holzbaur EL. 2007. Huntingtin facilitates dynein/dynactin-mediated vesicle transport. Proc Natl. Acad. Sci. U S A. 104(24): 10045-50

9. Chakrabarti O, and Hegde RS. 2009. Functional depletion of mahogunin by cytosolically exposed prion protein contributes to neurodegeneration. Cell 137(6): 1136-47

10. Colin E, Zala D, Liot G, Rangone H, Borrell-Pagès M, Li XJ, et al.. 2008. Huntingtin phosphorylation acts as a molecular switch for anterograde/retrograde transport in neurons. EMBO J. 27(15): 2124-34

11. Cota CD, Bagher P, Pelc P, Smith CO, Bodner CR, and Gunn TM. 2006. Mice with mutations in Mahogunin ring finger-1 (MGRN1) exhibit abnormal patterning of the leftright axis. Dev. Dyn. 235(12): 3438-47

12. Cox TC, Allen LR, Cox LL, Hopwood B, Goodwin B, Haan E, et al.. 2000. New mutations in MID1 provide support for loss of function as the cause of X-linked Opitz syndrome. Hum. Mol. Genet. 9(17): 2553-62 
13. Daniels MJ, Wang Y, Lee M and Venkitaraman AR. 2004. Abnormal cytokinesis in cells deficient in the breast cancer susceptibility protein BRCA2. Science 306(5697): 876-9

14. Delaval B, Ferrand A, Conte N, Larroque C, Hernandez-Verdun D, Prigent C, et al.. 2004. Aurora B-TACC1 protein complex in cytokinesis. Oncogene 23(26): 4516-22

15. Delgehyr N, Wieland U, Rangone H, Pinson X, Mao G, Dzhindzhev NS, et al.. 2006. Drosophila Mgr, a Prefoldin subunit cooperating with von HippelLindau to regulate tubulin stability. Proc. Natl. Acad. Sci. U S A 109(15): 5729-5734

16. Deshaies RJ and Joazeiro CA. 2009. RING domain E3 ubiquitin ligases. Annu. Rev. Biochem. 78, 399-434

17. Devenport D. 2014. The cell biology of planar cell polarity. J Cell Biol. 207(2):171-9

18. Dickins RA, Frew IJ, House CM, O'Bryan MK, Holloway AJ, Haviv I, et al.. 2002. The ubiquitin ligase component Siah1a is required for completion of meiosis I in male mice. Mol Cell Biol. 22(7): 2294-303

19. Ding Q, Lewis JJ, Strum KM, Dimayuga E, Bruce-Keller AJ, Dunn JC, et al.. 2002. Polyglutamine expansion, protein, aggregation, proteasome activity, and neural survival. J. Biol. Chem. 277(16): 13935-42

20. Du Q and Macara IG. 2004. Mammalian Pins is a conformational switch that links NuMA to heterotrimeric G proteins. Cell 119(4): 503-16

21. El-Hodiri HM, Shou W, and Etkin LD. 1997b. xnf7 functions in dorsal-ventral patterning of the Xenopus embryo. Dev. Biol. 190(1): 1-17

22. Elias S, Thion MS, Yu H, Sousa CM, Lasgi C, Morin X, et al.. 2014. Huntingtin Regulates Mammary Stem Cell Division and Differentiation. Stem Cell Report 2(4): 491-506

23. Funabiki H and Murray AW. 2000. The Xenopus chromokinesin Xkid is essential for metaphase chromosome alignment and must be degraded to allow anaphase chromosome movement. Cell 102(4): 411-24

24. Gao J, Huo L, Sun X, Liu M, Li D, Dong JT, et al.. 2008. The tumor suppressor CYLD regulates microtubule dynamics and plays a role in cell migration. J. Biol. Chem. 283(14): 8802-9

25. Germani A, Bruzzoni-Giovanelli H, Fellous A, Gisselbrecht S, Varin-Blank N, Calvo F. 2000. SIAH-1 interacts with $\alpha$-tubulin and degrades the kinesin Kid by the proteasome pathway during mitosis. Oncogene 19(52): 5997-6006 
26. Gillies TE and Cabernard C. 2011. Cell division orientation in animals. Curr. Biol. 21(15): R599-609

27. Godin JD, Colombo K, Molina-Calavita M, Keryer G, Zala D, Charrin BC et al.. 2010. Huntingtin Is Required for Mitotic Spindle Orientation and Mammalian Neurogenesis. Neuron 67(3): 392-406

28. Gönczy P. 2008. Mechanisms of asymmetric cell division: flies and worms pave the way. Nat. Rev. Mol. Cell Biol. 9(5): 355-66

29. Harhaj EW and Dixit VM. 2011. Deubiquitinases in the regulation of NF- $\kappa B$ signaling. Cell Res. 21(1): 22-39

30. He L, Lu XY, Jolly AF, Eldridge AG, Watson SJ, Jackson PK et al.. 2003. Spongiform degeneration in mahoganoid mutant mice. Science 299(5607): 710-2

31. Hertwig, O.1884. Das Problem der Befruchtung und der Isotropie des Eies. EineTheorie der Vererbung. Jenaische Zeitschriftfu" r Naturwissenschaft 18, 276-318.

32. Hoffner G, Kahlem P, and Djian P. 2002. Perinuclear localization of huntingtin as a consequence of its binding to microtubules through an interaction with beta-tubulin: relevance to Huntington's disease. J. Cell Sci. 115 (Pt 5): 941-8

33. House CM, Möller A, Bowtell DD. 2009. Siah proteins: novel drug targets in the Ras and hypoxia pathways. Cancer Res. 69(23): 8835-8838

34. Hristova VA, Beasley SA, Rylett RJ, and Shaw GS. 2009. Identification of a novel Zn2p-binding domain in the autosomal recessive juvenile Parkinson related E3 ligase parkin. J. Biol. Chem. 284(22): 14978-86

35. Jaakkola P, Mole DR, Tian YM, Wilson MI, Gielbert J, Gaskell SJ, et al.. 2001. Targeting of HIF-alpha to the von Hippel-Lindau ubiquitylation complex by O2regulated prolyl hydroxylation. Science 292(5516): 468-72

36. Jiao J, Kim HY, Liu RR, Hogan CA, Sun K, Tam LM, et al.. 2009. Transgenic analysis of the physiological functions of Mahogunin Ring Finger-1 isoforms. Genesis 47(8): 524-34

37. Joukov V, Groen AC, and Prokhorova T. 2006. The BRCA1/BARD1 Heterodimer Modulates Ran-Dependent Mitotic Spindle Assembly. Cell 127(3): 539-52

38. Kaelin Jr WG. 2008. The von Hippel-Lindau tumour suppressor protein: O2 sensing and cancer. Nat. Rev. Cancer. 8(11): 865-73

39. Kaelin WG. 2007. Von Hippel-Lindau disease. Annu. Rev. Pathol. 2,145-73

40. Kais Z, Chiba N, Ishioka C, and Parvin JD. 2012. Functional differences among BRCA1 missense mutations in the control of centrosome duplication. Oncogene 31(6): 799-804 
41. Kalchman MA, Graham RK, Xia G, Koide HB, Hodgson JG, Graham KC, et al.. 1996. Huntingtin is ubiquitinated and interacts with a specific ubiquitin-conjugating enzyme. J. Biol. Chem. 271(32): 19385-94

42. Kapitein LC, Peterman EJ, Kwok BH, Kim JH, Kapoor TM, and Schmidt CF. 2005. The bipolar mitotic kinesin Eg5 moves on both microtubules that it crosslinks. Nature 435(7038): $114-8$

43. Kim BY, Olzmann JA, Barsh GS, Chin LS, Li L. 2007. Spongiform neurodegenerationassociated E3 ligase Mahogunin ubiquitylates TSG101 and regulates endosomal trafficking. Mol. Biol. Cell. 18(4): 1129-42

44. Knoblich JA. 2008. Mechanisms of Asymmetric Stem Cell Division. Cell 132(4): 583-97

45. Kotak S, Busso C and Gönczy P. 2013. NuMA phosphorylation by CDK1 couples mitotic progression with cortical dynein function. EMBO J. 32(18): 2517-29

46. Lancaster OM and Baum B. 2011. Might makes right: Using force to align the mitotic spindle. Nat Cell Biol. 13(7): 736-8

47. Lin X, Liang $M$ and Feng XH. 2000. Smurf2 is a ubiquitin E3 ligase mediating proteasome-dependent degradation of Smad2 in transforming growth factor-beta signaling. J Biol. Chem. 275(47): 36818-22

48. Liu M, Aneja R, Sun X, Xie S, Wang H, Wu X et al.. 2008. Parkin regulates Eg5 expression by Hsp70 ubiquitination-dependent inactivation of c-Jun NH2- terminal kinase. J. Biol. Chem. 283(51): 35783-8

49. Liu W, Xin H, Eckert DT, Brown JA, Gnarra JR. 2011 .Hypoxia and cell cycle regulation of the von Hippel-Lindau tumor suppressor. Oncogene. 30(1): 21-31

50. Lu MS and Johnston CA. 2013. Molecular pathways regulating mitotic spindle orientation in animal cells. Development. 140(9): 1843-56

51. Maresca TJ, Niederstrasser H, Weis K, and Heald R. 2005. Xnf7 Contributes to Spindle Integrity through Its Microtubule-Bundling Activity. Curr. Biol. 15(19): 1755-61

52. Massoumi R. 2011. CYLD: A deubiquitination enzyme with multiple roles in cancer. Future Oncol. 7(2): 285-97

53. McGuire JR, Rong J, Li SH, and Li XJ. 2006. Interaction of Huntingtin associated protein-1 with kinesin light chain: implications in intracellular trafficking in neurons. J. Biol. Chem. 281(6): 3552-9

54. McGuire JR, Rong J, Li SH, and Li XJ. 2006. Interaction of Huntingtin-associated protein-1 with kinesin light chain: implications in intracellular trafficking in neurons. J. Biol. Chem. 281(6): 3552-9 
55. McNally FJ. 2013. Mechanisms of spindle positioning, J Cell Biol. 200(2): 131-40

56. Metzger MB, Hristova VA, Weissman AM. 2012. J Cell Sci. 125(Pt 3): 531-7

57. Miller KA, Gunn TM, Carrasquillo MM, Lamoreux ML, Galbraith DB and Barsh GS. 1997. Genetic Studies of the Mouse Mutations Mahogany and Mahoganoid. Genetics 146(4): $1407-15$

58. Molina-Calavita M, Barnat M, Elias S, Aparicio E, Piel M, Humbert S. 2014. Mutant huntingtin affects cortical progenitor cell division and development of the mouse neocortex. J. Neurosci. 34(30): 10034-40

59. Morin X and Bellaïche Y. 2011. Mitotic Spindle Orientation in Asymmetric and Symmetric Cell Divisions during Animal Development. Dev. Cell 21(1): 102-19

60. Nakayama K, Frew IJ, Hagensen M, Skals M, Habelhah H, Bhoumik A, et al.. 2004. Siah2 regulates stability of prolyl-hydroxylases, controls HIF1alpha abundance, and modulates physiological responses to hypoxia. Cell 117(7): 941-52

61. Narimatsu M, Bose R, Pye M, Zhang L, Miller B, Ching P, et al.. 2009. Regulation of Planar Cell Polarity by Smurf Ubiquitin Ligases. Cell 137(2): 295-307

62. Nasir J, Floresco SB, O'Kusky JR, Diewert VM, Richman JM, Zeisler J et al.. 1995. Targeted disruption of the Huntington's disease gene results in embryonic lethality and behavioral and morphological changes in heterozygotes. Cell 81(5): 811-23

63. Neumüller RA and Knoblich JA. 2009. Dividing cellular asymmetry: asymmetric cell division its implications for stem cells and cancer. Genes Dev. 23(23): 2675-99

64. Osmundson EC, Ray D, Moore FE, and Kiyokawa H. 2009. Smurf2 as a novel mitotic regulator: From the spindle assembly checkpoint to tumorigenesis. Cell div. 4(14) doi: $10.1186 / 1747-1028-4-14$

65. Parvin JD. 2009. The BRCA1-dependent ubiquitin ligase, gamma-tubulin, and centrosomes. Environ. Mol. Mutagen. 50(8): 649-53

66. Peng CY, Manning L, Albertson R and Doe CQ. 2000. The tumour-suppressor genes lgl and dlg regulate basal protein targeting in Drosophila neuroblasts. Nature 408(6812): 596-600

67. Ryser S, Dizin E, Jefford CE, Delaval B, Gagos S, Christodoulidou A, Krause KH et al.. 2009. Distinct roles of BARD1 isoforms in mitosis: full-length BARD1 mediates Aurora B degradation, cancer-associated BARD1beta scaffolds Aurora B and BRCA2. Cancer Res. 69(3): 1125-34 
68. Sankaran S, Starita LM, Groen AC, Ko MJ, and Parvin JD. 2005. Centrosomal microtubule nucleation activity is inhibited by BRCA1-dependent ubiquitination. Mol. Cell Biol. 25(19): 8656-68

69. Sawin KE and Mitchison TJ. 1995. Mutations in the kinesin-like protein Eg5 disrupting localization to the mitotic spindle. Proc. Natl. Acad. Sci. U S A. 92(10): 4289-93

70. Schober M, Schaefer M and Knoblich JA. 1999. Bazooka recruits Inscuteable to orient asymmetric cell divisions in Drosophila neuroblasts. Nature 402(6761): 548-51

71. Setsuie R and Wada K. 2007. The functions of UCH-L1 and its relation to neurodegenerative diseases. Neurochem. Int. 51(2-4): 105-11

72. Sharp DJ, Yu KR, Sisson JC, Sullivan W, Scholey JM. 1999. Antagonistic microtubulesliding motors position mitotic centrosomes in Drosophila early embryos. Nat. Cell Biol. 1(1): $51-4$

73. Siller HK and Doe CQ, 2009. Spindle orientation during asymmetric cell division. Nat. Cell Biol. 11(4): 365-74

74. Simons M and Mlodzik M. 2008. Planar cell polarity signaling: from fly development to human disease. Annu Rev Genet. 42:517-40

75. Srivastava D and Chakrabarti O. 2014. Mahogunin-mediated $\alpha$-tubulin ubiquitination via noncanonical K6 linkage regulates microtubule stability and mitotic spindle orientation. Cell Death Dis. 5:e1064. doi: 10.1038/cddis.2014.1

76. Stevermann L and Liakopoulos D. 2012. Molecular mechanisms in spindle positioning: structures and new concepts. Curr. Opin. Cell Biol. 24(6): 816-24

77. Stieglitz B, Morris-Davies AC, Koliopoulos MG, Christodoulou E, Rittinger K. 2012. LUBAC synthesizes linear ubiquitin chains via a thioester intermediate. EMBO Rep. 13(9): 840-6

78. Stremlau M, Owens CM, Perron MJ, Kiessling M, Autissier P, Sodroski J. 2004. The cytoplasmic body component TRIM5 alpha restricts HIV-1 infection in Old World monkeys. Nature, 427(6977): 848-53

79. Sun X, Liu M, Hao J, Li D, Luo Y, Wang X, et al.. 2013. Parkin deficiency contributes to pancreatic tumorigenesis by inducing spindle multipolarity and misorientation. Cell Cycle 12(7): 1133-1141

80. Tanenbaum ME and Medema RH. 2010. Mechanisms of centrosome separation and bipolar spindle assembly. Dev. Cell. 19(6): 797-806 
81. Tanenbaum ME, Macůrek L, Janssen A, Geers EF, Alvarez-Fernández M, Medema RH. 2009. Kif15 cooperates with eg5 to promote bipolar spindle assembly. Curr. Biol. 19(20):1703-11

82. Tang LY, Yamashita M, Coussens NP, Tang Y, Wang X, Li C, Deng CX et al.. 2011. Ablation of Smurf2 reveals an inhibition in TGF- $\beta$ signaling through multiple monoubiquitination of Smad3. EMBO J. 30(23): 4777-89

83. Tarapore P, Hanashiro K and Fukasawa K. 2012. Analysis of centrosome localization of BRCA1 and its activity in suppressing centrosomal aster formation. Cell Cycle 11(15): 2931-46.

84. Thoma CR, Toso A, Gutbrodt KL, Reggi SP, Frew IJ, Schraml P et al.. 2009. VHL loss causes spindle misorientation and chromosome instability. Nat. Cell Biol. 11(8) 9941001

85. vanHeesbeen RG, Tanenbaum ME and Medema RH. 2014. Balanced Activity of Three Mitotic Motors Is Required for Bipolar Spindle Assembly and Chromosome Segregation. Cell Reports. 8(4): 948-56

86. Waters JC, Cole RW and Rieder CL. 1993. The force-producing mechanism for centrosome separation during spindle formation in vertebrates is intrinsic to each aster. $\mathrm{J}$. Cell Biol. 122(2): 361-72

87. Wenzel DM, Lissounov A, Brzovic PS, Klevit RE. 2011. UBCH7 reactivity profile reveals parkin and HHARI to be RING/HECT hybrids. Nature. 474(7349): 105-8

88. Whitehead CM, Winkfein RJ and Rattner JB. 1996. The relationship of HsEg5 and the actin cytoskeleton to centrosome separation. Cell Motil Cytoskeleton. 35(4): 298-308

89. Williams SE and Fuchs E. 2013. Oriented divisions, fate decisions. Curr. Opin. Cell Biol. 25(6): 749-58

90. Wodarz A, Ramrath A, Grimm A and Knust E. 2000. Drosophila atypical protein kinase $\mathrm{C}$ associates with Bazooka and controls polarity of epithelia and neuroblasts. J Cell Biol. 150(6): 1361-74

91. Wu LC, Wang ZW, Tsan JT, Spillman MA, Phung A, Xu XL et al.. 1996. Identification of a RING protein that can interact in vivo with the BRCA1 gene product. Nat. Genet. 14(4): 430-40

92. Yang Y, Liu M, Li D, Ran J, Gao J, Suo S, et.al.. 2014. CYLD regulates spindle orientation by stabilizing astral microtubules and promoting dishevelled-NuMA-dynein/ dynactin complex formation. Proc. Natl. Acad. Sci. 111(6): 2158-63 
93. Zeitlin S, Liu JP, Chapman DL, Papaioannou VE, and Efstratiadis A. 1995. Increased apoptosis and early embryonic lethality in mice nullizygous for the Huntington's disease gene homologue. Nat. Genet. 11(2): 155-63

94. Zhang C, Hughes M and Clarke PR. 1999. Ran-GTP stabilises microtubule asters and inhibits nuclear assembly in Xenopus egg extracts. J Cell Sci. 112 ( t 14): 2453-61

95. Zhang J, Wang Y, Gao Z, Yun Z, and Ye J. 2012. Hypoxia-inducible Factor 1 Activation from aP2 (Fabp4)-Cre Mediated Knockout of Von Hippel-Lindau Gene Leads to Embryonic Lethality. Clin. Exp. Pharmacol. Physiol. 39(2): 145-50 


\section{Figure Legends}

Figure 1: Types of cell division in the various cell types in the body. (a) Schematic diagram showing the relevance of symmetric/asymmetric division in stem cells. (b) This shows one of the possible ways by which external cues could cause erroneous spindle positioning in the cell leading to asymmetric division in non-polarized cells. Internal cues, like altered distribution of the cortical regulators could also give rise to a similar situation. (c) Symmetric and asymmetric division in case of polarized cells is shown. While the former is seen along the anterior -posterior axis, division along the apico-basal axis leads to asymmetric cell division. (d) An overview of different the cortical players important for spindle orientation. LGN acts as an adaptor protein which holds NuMa and $\mathrm{G}_{\mathrm{i}}$. This ternary complex recruits dynein at the cell cortex by the help of motor proteins. NuMa helps establish a cortical zone enriched in dynein by binding to it. Dynein then binds the astral microtubules and regulates spindle positioning in cells. Polo Kinase 1 present on spindles serves to negatively regulate the cortical dynein localization while the RanGTP gradient present on the chromosomes negatively regulates $\mathrm{NuMa} / \mathrm{LGN}$ distribution in the lateral cell cortex. It is the cooperative balance of these two gradients that positions the cortical proteins in the right place, thus ensuring correct spindle positioning. Further, players like CLASP1 (not shown in the figure) belonging to a family of Microtubule Associated Proteins (MAPs), are involved in the correct capture of the astral microtubules and their attachment at the cell cortex.

Figure 2: PARK2 and spindle defects. Schematic of the possible mode of action of PARK2 by which it affects spindles and causes spindle multipolarity.

Figure 3: BRCA1: centrosomal and spindle defects. Summary of the mode of action of BRCA1 by which it affects spindles and causes genomic instability.

Figure 4: MGRN1 and spindle tilt. An overview of the effect of MGRN1 on spindles and its plausible mode of action.

Figure 5: A summary of the different target proteins/ structures regulated by ubiquitination in the cortex of the cell during mitosis. Ligases like MGRN1, VHL, Xnf7 and SIAH1 regulate microtubule assembly or polymerization, while BRCA1/BARD1 regulates the centrosomal apparatus. PARK2 and HTT affect microtubule motors, thus in turn regulating the correct localization of the cortical effectors of mitosis. 

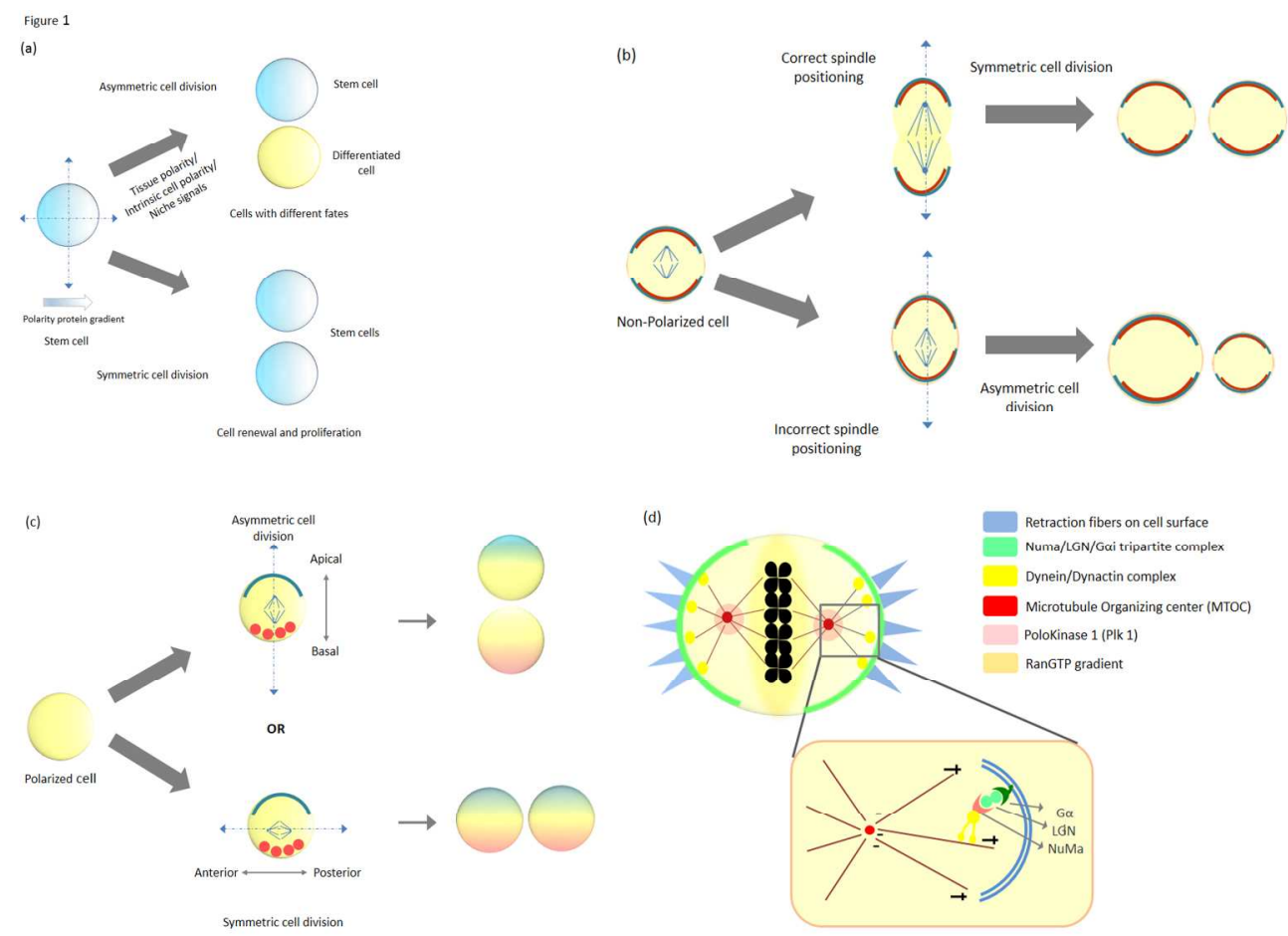

Types of cell division in the various cell types in the body. $229 \times 165 \mathrm{~mm}$ ( $300 \times 300$ DPI) 
Figure 2

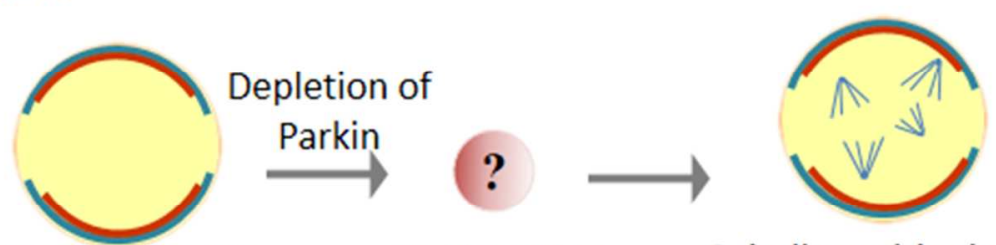

Wild Type PARK2

Unidentified Spindle multipolarity, effector molecule

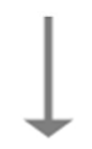

Increased multimonoubiquitination of $\mathrm{Hsp} 70$

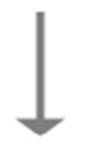

Activation of c-Jun $\mathrm{NH}$ 2-terminal kinase

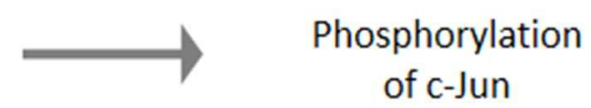

PARK2 and spindle defects.

$106 \times 76 \mathrm{~mm}(300 \times 300$ DPI $)$ 
Figure 3

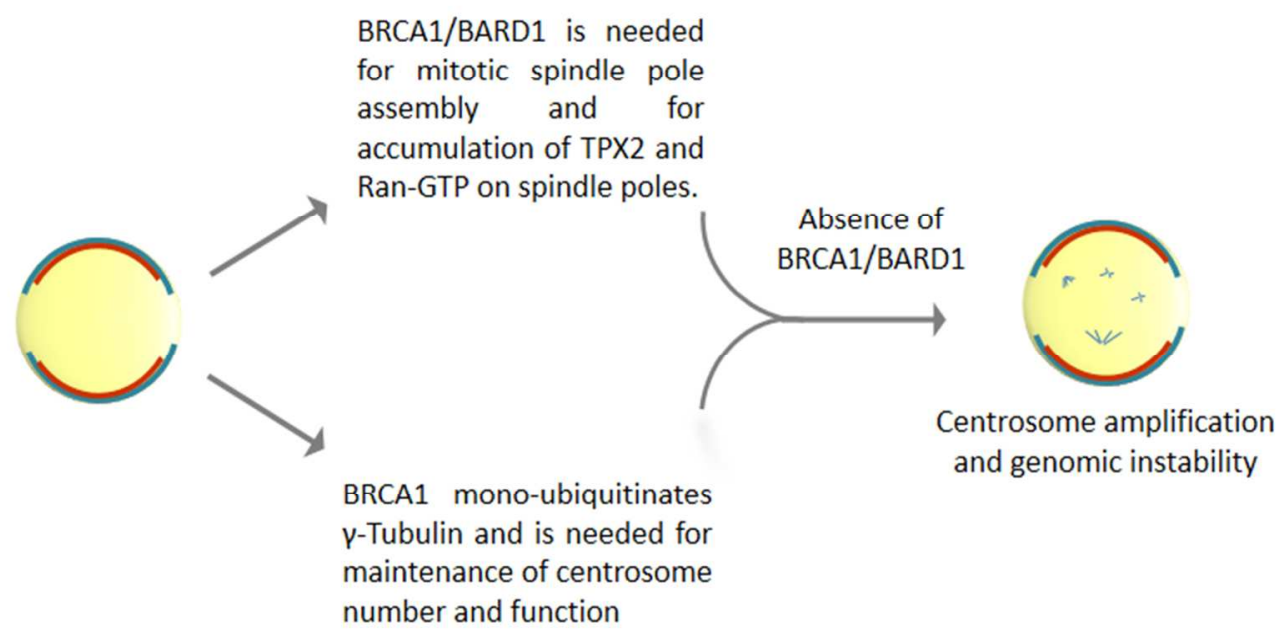

BRCA1: centrosomal and spindle defects.

$143 \times 76 \mathrm{~mm}(300 \times 300 \mathrm{DPI})$ 
Figure 4

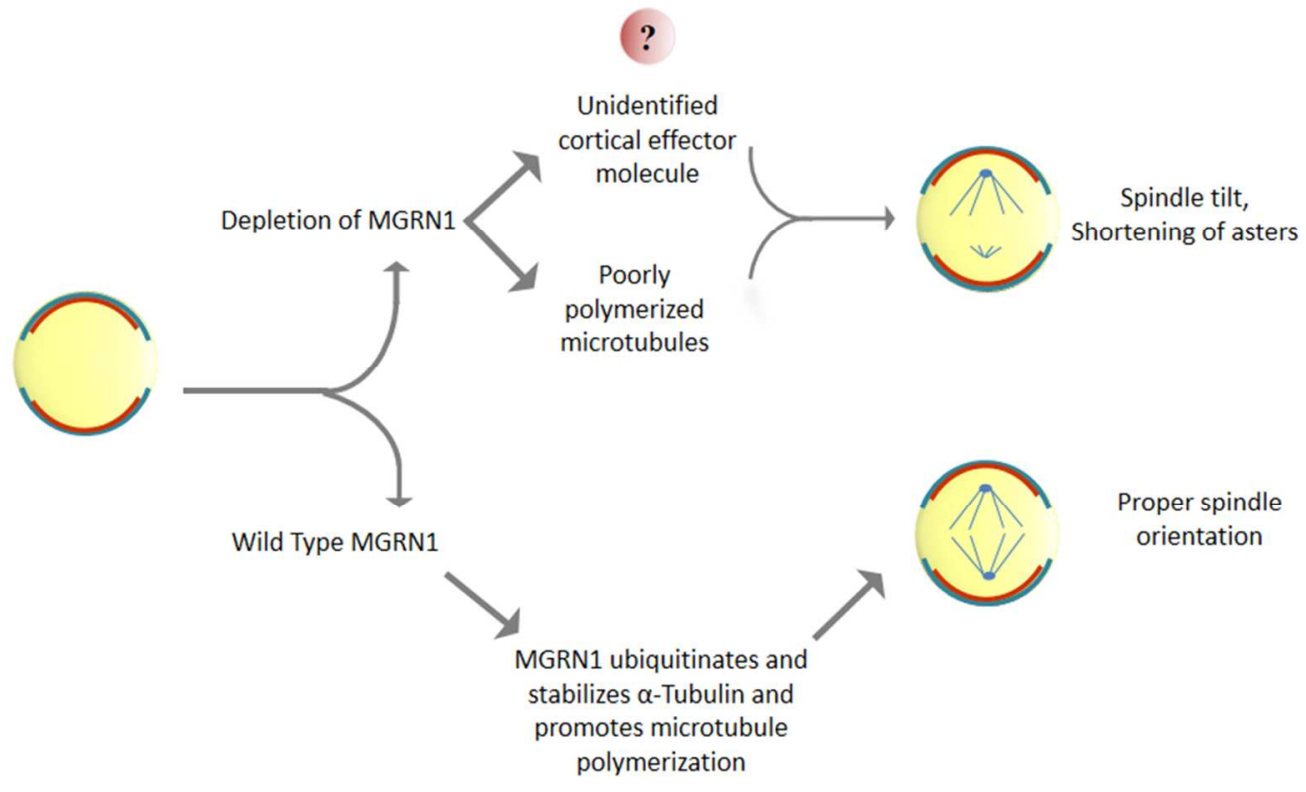

MGRN1 and spindle tilt.

$120 \times 76 \mathrm{~mm}$ (300 x 300 DPI) 


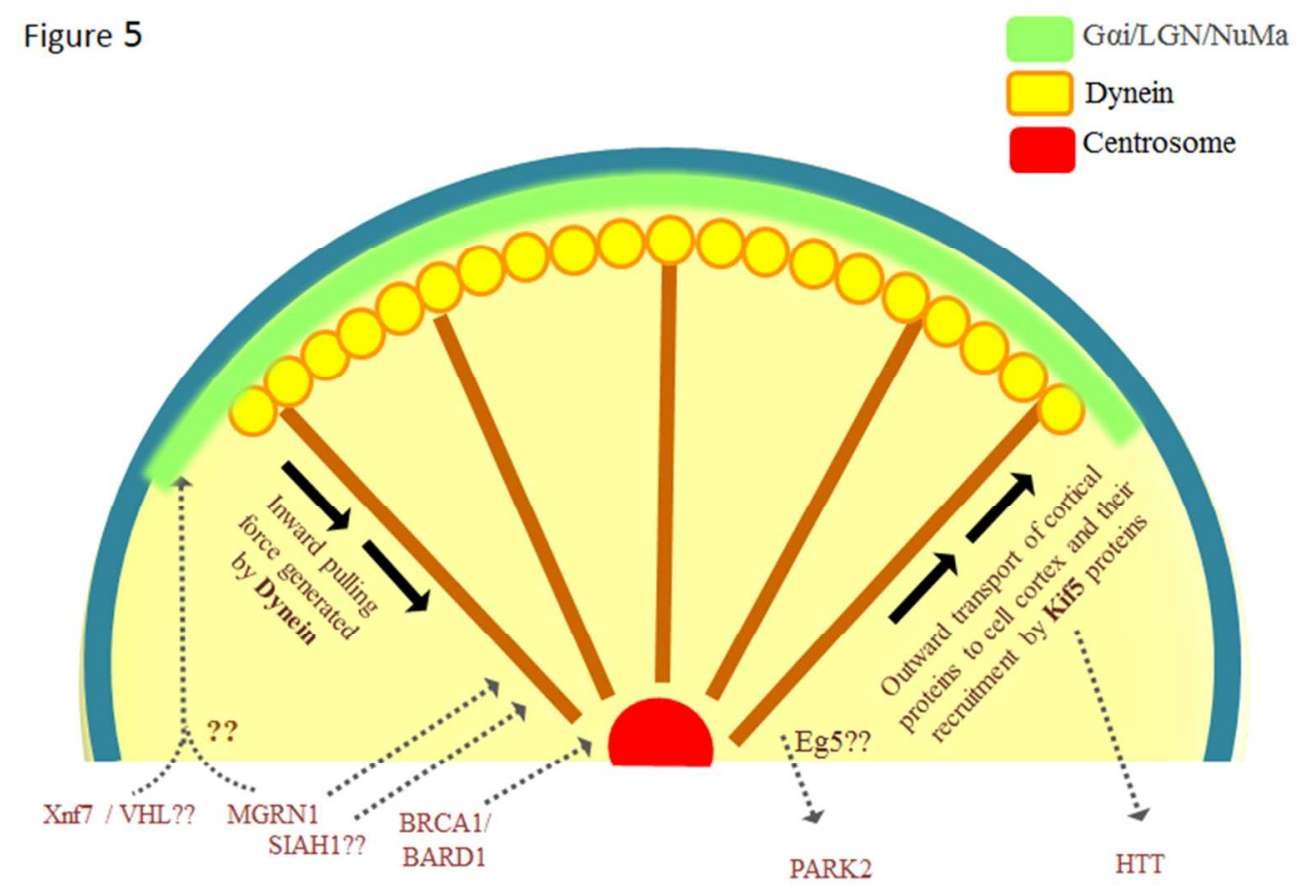

A summary of the different target proteins/ structures regulated by ubiquitination in the cortex of the cell during mitosis. $110 \times 76 \mathrm{~mm}(300 \times 300$ DPI $)$ 